\title{
Comparative and Quantitative Study of Fundamental Approaches on Digital Aerial Image Deblurring
}

\author{
Zhengmao Ye and Habib Mohamadian
}

\begin{abstract}
Digital image deblurring is a classical real-world problem with applications ranging from astronomy to photography. For aerial digital images, image blurring is inevitable due to object moving, platform moving and possible camera shaking. Image deblurring is to estimate the actual scene by sharpening the observed images being blurred or corrupted by any motion. At the same time, it aims to retain important object features as much as possible. It plays an important role in information decision support systems. There are many numerical filtering, deconvolution and optimization approaches to sharpen the blurry images. Certain approaches even combine with implementation of artificial intelligence. Some of them are in fact very complicated in theory and practice. This article applies several fundamental methods to aerial image deblurring. Satisfactory results on each level of true color aerial images are obtained with ease using these fundamental technologies. In addition, quantitative measures are proposed to evaluate the outcomes in an objective manner, where metrics of discrete entropy, relative entropy, discrete energy, mutual information, contrast and homogeneity are introduced for evaluating aerial image deblurring. This approach has the potential for accurate decision making.
\end{abstract}

Index Terms - Image deblurring, deconvolution, filtering, optimization, quantitative analysis.

\section{INTRODUCTION}

Processing of the aerial images has broad applications in the fields of space exploration, ground surveillance, national defense, target detection, environmental exploration and weather forecasting, and so on. Any elevated platform will be appropriated for the aerial photo taking. However, these images are affected by multiple factors, such as atmospheric medium dispersion, severe weather conditions and diverse types of illumination, pressure and temperature, etc. For instance, the poorer the illumination, the higher the blurring. Thus, the quality of aerial images might be significantly affected, giving rise to the needs of image deblurring [1]-[5]. A statistical method of blind deconvolution is presented for deblurring images with two unknown gray levels. The basic idea is to adjust a deblurring filter until its output becomes two tones. By minimizing optimization criteria, the original image, along with the filter, will be recovered uniquely at the high SNR. Without prior knowledge, it can recover even correlated and non-stationary images [6].

Another nonparametric regression method is proposed for deblurring noisy images. The algorithm is nonlinear and spatially adaptive with respect to both smoothness and

Manuscript received November 16, 2012; revised March 23, 2013

Z. Ye is with Southern University, Baton Rouge, Louisiana 70813, USA (e-mail: zhengmao_ye@subr.edu).

H. Mohamadian is with Southern University, Baton Rouge, Louisiana 70813, USA (e-mail: habib_mohamadian@subr.edu). irregularities of corrupted images. Multi-resolution wavelet algorithms produce estimations which are combined from different scale projections. Regularized inverse and Wiener inverse filters serve as deblurring operators used jointly with directional kernel filters. It outperforms some of the best existing methods [7]. With an extension of a least-squares estimation method, the multi-image deblurring is reduced to the computationally efficient single-image deblurring. Via stable operations on normal equations, the convergence rate of iterative algorithms has been speeded up [8]. A unique mathematical framework is proposed to deblur, denoise and compress natural images. Images are decomposed in a wavelet packet basis adapted to both the deblurring filter and denoising process. Effective denoising is performed by thresholding small coefficients while deblurring is obtained by multiplying the coefficients with a deconvolution kernel. The representation is compressed by quantizing remaining coefficients and by coding values using a context-based entropy coder. The treatments are successfully applied to the satellite image chain [9]. For blurred and noisy satellite images, a hybrid thresholding technique can lead to high quality results, which exhibit both correctly restored textures and high signal to noise ratios in the homogeneous areas. Usage of complex wavelet packets enables the translational invariance and improves directional selectivity. It improves restoration since it is faster, more rotationally invariant and better taking into account the directions of the details and textures of the image [10]. The new image denoising method based on Wiener filtering for soft thresholding has been proposed. It shows a high and stable signal to noise ratio gain for all noise models used. This process leads to an improvement of phase images when real and imaginary parts of the wavelet packet coefficients are independently filtered [11]. The particle swarm optimization technique is also proposed for image deblurring. The degraded image is taken as particle and a population of images is generated to build up a swarm of variable size. This approach is validated on real images degraded by a Gaussian blurring and an additive Gaussian noise [12].

It is shown that classical and modern filtering approaches can both be successfully applied to image deblurring. Thus, computational complexity is another important factor to be considered. For example, the approaches of wavelet packets and particle swarm intelligence will improve the quality of image with a tradeoff in the computation costs. In this case, the quantitative approach is also proposed to evaluate some fundamental image deblurring techniques, rather than visual appealing, which can be easily expanded to other filtering approaches [4], [13]-[15]. For the gray level images, metrics of discrete entropy, relative entropy, discrete energy, mutual information, contrast and homogeneity have been introduced 
for objective measurement. In the true color system, similar concepts can be implemented as well at each of three color levels. The discrete entropy, relative entropy, mutual information, discrete energy, mutual information, contrast and homogeneity of three color component images could serve as statistical measures of randomness, which are used to characterize the source and deblurred images.

\section{FUNDAMENTAL SCHEMES FOR IMAGE DEBLURRING}

\section{A. Blind Deconvolution Deblurring}

The blind deconvolution is a traditional technique to recover the target image from a set of blurred images with no information available on image distortion (blurring and noisy). It can restore the image and unknown point spread function (PSF) at the same time. The PSF is estimated from the image set, allowing the deconvolution to be performed. The proposed speedup blind deconvolution is performed iteratively via Richardson-Lucy deconvolution algorithms. It improves the estimation of the PSF and the scene in each iteration. The trial and error approach is used herein to determine the desirable threshold level and additional PSF constraints can be selected using zero padding. In the frequency domain, the blind deconvolution is expressed as (1). Based on image observation, the degradation function can be estimated as (2), where the tilde $\mathrm{F}\left(\omega_{1}, \omega_{2}\right)$ represents the Fourier transform of estimation of the true image $\mathrm{F}(x, y)$, $\mathrm{G}\left(\omega_{1}, \omega_{2}\right)$ represents the Fourier transform of the blurred image, $\mathrm{H}\left(\omega_{1}, \omega_{2}\right)$ represents the degradation function, and $k$ is a coefficient to be adjusted.

$$
\begin{gathered}
\tilde{\mathrm{F}}\left(\omega_{1}, \omega_{2}\right)=\mathrm{G}\left(\omega_{1}, \omega_{2}\right) / \mathrm{H}\left(\omega_{1}, \omega_{2}\right) \\
\mathrm{H}\left(\omega_{1}, \omega_{2}\right)=\mathrm{e}^{-\mathrm{k}\left(\omega_{1}^{2}+\omega_{2}^{2}\right)^{1 / 2}}
\end{gathered}
$$

\section{B. Constrained Linear Least Squares Deblurring}

Constrained linear least-squares optimization can also be used to recover the blurred images. The objective is to minimize the Euclidean vector norm of the estimation error between the blurred image and estimated true image. The solution in the frequency domain is given by (3):

$$
\tilde{\mathrm{F}}\left(\omega_{1}, \omega_{2}\right)=\mathrm{G}\left(\omega_{1}, \omega_{2}\right) \frac{\mathrm{H}^{*}\left(\omega_{1}, \omega_{2}\right)}{\left|\mathrm{H}\left(\omega_{1}, \omega_{2}\right)\right|^{2}+k\left|\mathrm{~L}\left(\omega_{1}, \omega_{2}\right)\right|^{2}}
$$

where the tilde $F\left(\omega_{1}, \omega_{2}\right)$ represents the Fourier transform of estimation of the true image $F(x, y), G\left(\omega_{1}, \omega_{2}\right)$ represents the Fourier transform of the blurred image, $\mathrm{H}\left(\omega_{1}, \omega_{2}\right)$ represents the degradation function and $\mathrm{H}^{*}\left(\omega_{1}, \omega_{2}\right)$ is the complex conjugate of $\mathrm{H}\left(\omega_{1}, \omega_{2}\right), \mathrm{L}\left(\omega_{1}, \omega_{2}\right)$ is the Fourier transform of the Laplacian operator, and $\mathrm{k}$ is a coefficient to be adjusted.

The least squares algorithm can be simply depicted in the spatial domain.

$$
\operatorname{MIN}\left\{\|\mathrm{H}(x, y) \mathrm{F}(x, y)-\mathrm{G}(x, y)\|^{2}+\mathrm{k}\|\mathrm{L}(x, y) \mathrm{F}(x, y)\|^{2}\right\}
$$

where $\mathrm{F}(x, y)$ is the true image to be determined, $\mathrm{H}(x, y)$ is the degradation operator with the constraints within an interval $[0,1], \mathrm{G}(x, y)$ is the blurred image, and $\mathrm{L}(x, y)$ is the discrete Laplacian, which associates each pixel to those surrounding it. It can be simplified into (5) via zero padding.

$$
\operatorname{MIN}\left\{\|\mathrm{A}(x, y) \mathrm{F}(x, y)-\mathrm{B}(x, y)\|^{2}\right\}
$$

where the matrix $\mathrm{A}(x, y)$ combines $\mathrm{H}(x, y)$ and $\mathrm{k}^{1 / 2} \mathrm{~L}(x, y)$, and the matrix $\mathrm{B}(x, y)$ combines $\mathrm{G}(x, y)$ and zero matrix to match the dimension.

The optimal solution is calculated via pseudo-inverse:

$$
\mathrm{F}(x, y)=\left(\mathrm{A}^{*} \mathrm{~A}\right)^{-1} \mathrm{~A}^{*} \mathrm{~B}
$$

where $\mathrm{A}^{*}$ is the complex conjugate of matrix A. Selected constraints guarantee that $\mathrm{A}^{*} \mathrm{~A}$ is perfectly well conditioned.

\section{Wiener Filter Deblurring}

A blurred image can be modeled by $2 \mathrm{D}$ convolution of the source image and a low pass filter. In order to restore the source image, the blurred image should convolute with a type of high pass filter. In ideal cases, the high pass filter can be determined by inverting all elements of discrete Fourier transform (DFT) of the low pass filter. In order to avoid near zero values to be inversed, a suitable threshold is necessary. Essentially an inverse filter is a high pass filter which is sensitive to noises, in this case, the Wiener filter is selected for image deblurring, which is a natural extension of the inverse filter when noises are present. A simplified expression of the Wiener filter in the frequency domain is expressed as (7):

$$
\tilde{\mathrm{F}}\left(\omega_{1}, \omega_{2}\right)=\frac{\mathrm{G}\left(\omega_{1}, \omega_{2}\right)}{\mathrm{H}\left(\omega_{1}, \omega_{2}\right)}\left[\frac{\left|\mathrm{H}\left(\omega_{1}, \omega_{2}\right)\right|^{2}}{\left|\mathrm{H}\left(\omega_{1}, \omega_{2}\right)\right|^{2}+k}\right]
$$

where the tilde $\mathrm{F}\left(\omega_{1}, \omega_{2}\right)$ represents the Fourier transform of estimation of the true image $\mathrm{F}(x, y), \mathrm{G}\left(\omega_{1}, \omega_{2}\right)$ represents the Fourier transform of the blurred image, and $\mathrm{H}\left(\omega_{1}, \omega_{2}\right)$ represents the degradation function. As the power spectrum of the true image $\mathrm{F}(x, y)$ is unknown, $\mathrm{k}$ is introduced as a specific constant via the trial and error to represent the Noise-to-Signal ratio.

\section{CASE Studies On IMAGe DebluRring}

The first aerial image selected is an ocean-island view near Puerto Rico and the second aerial image selected is a mountain-snow view in northern Canada, as shown in Figs. 1-2, where the source images are followed by deblurred images using three deblurring schemes, respectively.
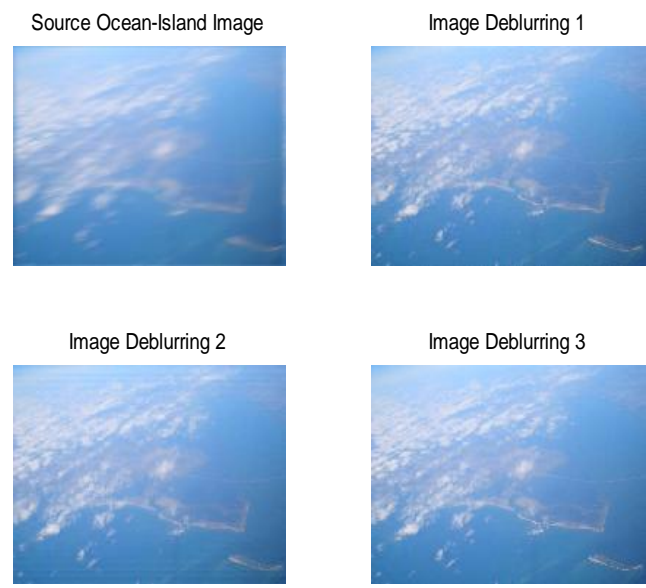

Image Deblurring 3

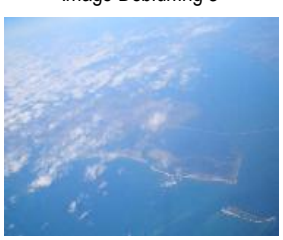

Fig. 1. Source Ocean-Island View and Deblurred Images 

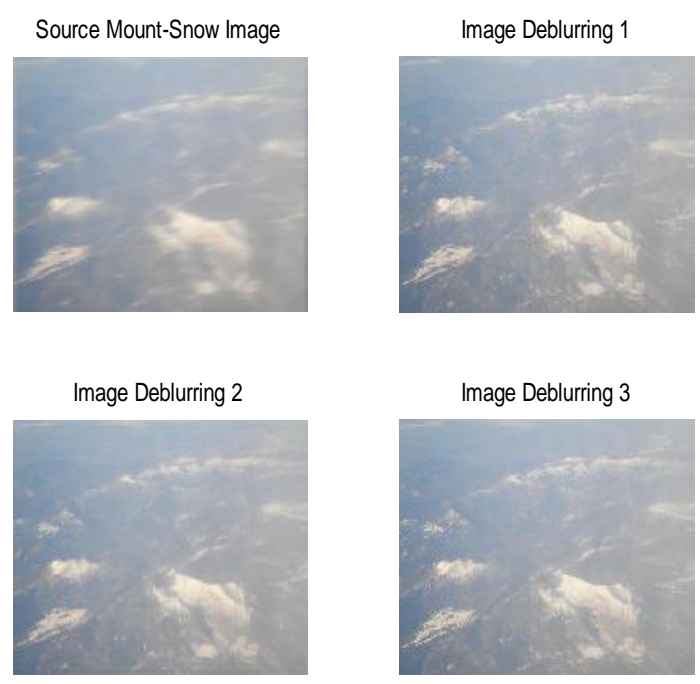

Fig. 2. Source Mount-Snow Image and Deblurred Images

In Fig. 3, the true color source blurry images are represented in three levels of red, green and blue. In Figs. 46 , the three level deblurred images are shown after using three fundamental deblurring schemes: (1) Blind deconvolution; (2) Linear least squares estimation; (3) Wiener Filtering. In Figs. 3-6, results of the image one are listed in the first row and results of the image two are listed in the second row. Image quality enhancement and some differences in appearance could be slightly observed. However, objective measures should be further applied to evaluate the outcomes quantitatively.

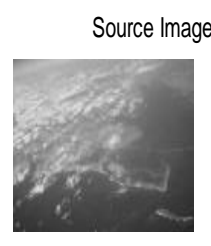

Red Level

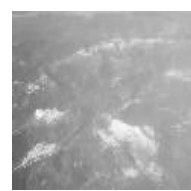

Fig. 3. Source Images in Red, Green and Blue Levels ( $1^{\text {st }}$ Row: Source Image $1 ; 2^{\text {nd }}$ Row: Source Image 2$)$

Deblurring Scheme \#1 Displayed in Red, Green and Blue Levels
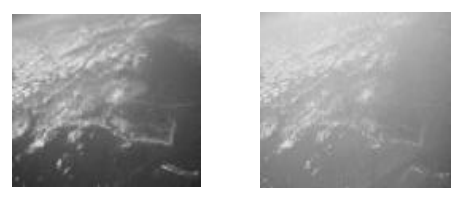

Green Level

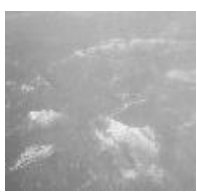

Blue Level

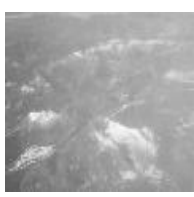

Fig. 4. Image Deblurring via Blind Deconvolution ( $1^{\text {st }}$ Row: Deblurred Image $1 ; 2^{\text {nd }}$ Row: Deblurred Image 2)

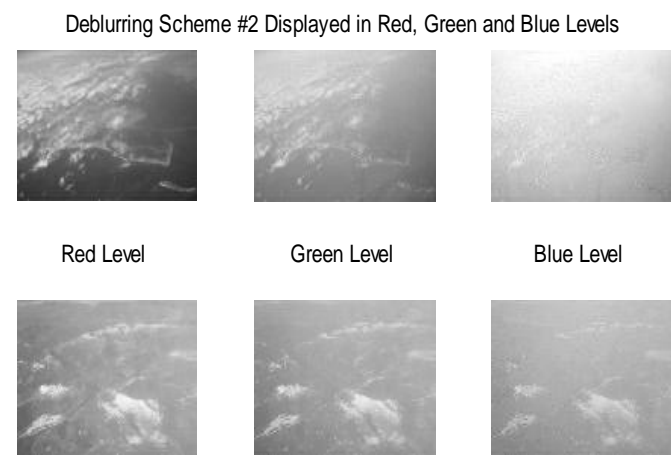

Fig. 5. Deblurring via Least Squares Estimation ( $1^{\text {st }}$ Row: Deblurred Image $1 ; 2^{\text {nd }}$ Row: Deblurred Image 2 )

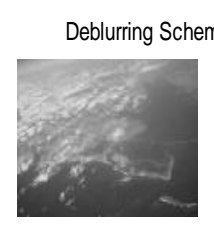

Red Level

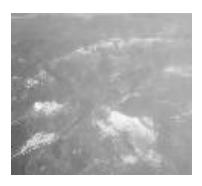

Fig. 6. Deblurring via Wiener Filtering ( $1^{\text {st }}$ Row: Deblurred Image $1 ; 2^{\text {nd }}$ Row: Deblurred Image 2 )

\section{QUANTITATIVE ANALYSIS}

\section{A. Histogram and Probability Functions}

A digital image with $\mathrm{M}$ by $\mathrm{N}$ pixels has been considered. Occurrence of each color level is described as the cooccurrence matrix of relative frequencies. The occurrence probability function is then estimated from its histogram, which is formulated as (8):

$$
p(k)=\frac{h(k)}{\sum h(k)}
$$

where $p(k)$ is the probability distribution function and $h(k)$ is the histogram function.

\section{B. Discrete Entropy in Image Deblurring}

The discrete entropy is a measure of information content, which can be interpreted as the average uncertainty of the information source. The discrete entropy is the summation of the products of the probability of the outcome multiplied by the logarithm of the inverse of probability of the outcome, considering all possible outcomes $\{1,2, \ldots, n\}$ at each color level in the event set $\left\{x_{1}, x_{2}, \ldots, x_{n}\right\}$, where $p(i)$ is the probability at the level $i$, which contains all the histogram counts. The discrete entropy $\mathrm{H}(x)$ is formulated as (9) whose corresponding results are shown in Table I.

$$
\mathrm{H}(x)=\sum_{i=1}^{k} p(i) \log _{2} \frac{1}{p(i)}=-\sum_{i=1}^{k} p(i) \log _{2} p(i)
$$

From Table I, it is shown that for each color level, all three deblurring schemes actually produce sharper images 
containing more information than the blurry source images.

TABLE I: DISCRETE ENTROPY IN IMAGE DEBLURRING

\begin{tabular}{lccc}
\hline Discrete Entropy & Red Level & Green Level & Blue Level \\
\hline Source Ocean Image & 7.3265 & 6.8555 & 6.6397 \\
Deblurring Scheme 1 & 7.3478 & 6.8813 & 6.6561 \\
Deblurring Scheme 2 & 7.3380 & 6.8703 & 6.6557 \\
Deblurring Scheme 3 & 7.3517 & 6.8942 & 6.6512 \\
Source Mount Image & 6.5319 & 6.2180 & 5.9569 \\
Deblurring Scheme 1 & 6.5509 & 6.2534 & 6.0095 \\
Deblurring Scheme 2 & 6.5383 & 6.2398 & 5.9910 \\
Deblurring Scheme 3 & 6.5588 & 6.2590 & 6.0237 \\
\hline
\end{tabular}

\section{Relative Entropy in Image Deblurring}

Suppose two discrete probability distributions of digital images have the probability functions of $p(i)$ and $q(i)$. The relative entropy of one with respect to another is then defined as the summation of all the possible states of the system, which is formulated as (10). The relative entropies of two source images and deblurred images are shown in Table II.

$$
d=\sum_{i=1}^{k} p(i) \log _{2} \frac{p(i)}{q(i)}
$$

From Table II, it is shown that the least squares estimation (Scheme 2) provides better information match between the source blurry image and sharpened images than those of the Wiener filtering and blind deconvolution.

\section{TABLE II: RELATIVE ENTROPY IN IMAGE DEBLURRING}

\begin{tabular}{lccc}
\hline Relative Entropy & Red Level & Green Level & Blue Level \\
\hline Source Ocean Image & & & \\
Deblurring Scheme 1 & 0.0072 & 0.0109 & 0.0087 \\
Deblurring Scheme 2 & 0.0085 & 0.0116 & 0.0093 \\
Deblurring Scheme 3 & 0.0064 & 0.0081 & 0.0037 \\
Source Mount Image & & & \\
Deblurring Scheme 1 & 0.0074 & 0.0116 & 0.0113 \\
Deblurring Scheme 2 & 0.0092 & 0.0127 & 0.0139 \\
Deblurring Scheme 3 & 0.0035 & 0.0041 & 0.0072 \\
\hline
\end{tabular}

\section{Mutual Information in Image Deblurring}

Another metric of the mutual information $I(X ; Y)$ can be also applied, which is to describe how much information one variable tells about the other variable. The mutual information is formulated as (11).

$$
\begin{aligned}
& I(X ; Y)=\sum_{X, Y} p_{X Y}(X, Y) \log _{2} \frac{p_{X Y}(X, Y)}{p_{X}(X) p_{Y}(Y)} \\
& =-\sum_{X} p_{X}(X) \log _{2} p_{X}(X)+\sum_{X, Y} p_{X Y}(X, Y) \log _{2} \frac{p_{X Y}(X, Y)}{p_{Y}(Y)} \\
& =H(X)-H(X \mid Y)
\end{aligned}
$$

where $H(X)$ and $H(X \mid Y)$ are values of the entropy and conditional entropy; $p_{X Y}$ is the joint probability density function; $p_{X}$ and $p_{Y}$ are the marginal probability density functions. It can be interpreted as information that $Y$ can tell about $X$ is the reduction in uncertainty of $X$ due to the existence of $Y$. It can also be regarded as the relative entropy between the joint distribution and product distribution. Results of mutual information between the original and deblurred images are shown in Table III.

TABLE III: MUTUAL INFORMATION IN IMAGE DEBLURRING

\begin{tabular}{lccc}
\hline Mutual Information & Red Level & Green Level & Blue Level \\
\hline Source Ocean Image & & & \\
Deblurring Scheme 1 & 0.0213 & 0.0258 & 0.0164 \\
Deblurring Scheme 2 & 0.0115 & 0.0148 & 0.0161 \\
Deblurring Scheme 3 & 0.0252 & 0.0387 & 0.0115 \\
Source Mount Image & & & \\
Deblurring Scheme 1 & 0.0190 & 0.0354 & 0.0526 \\
Deblurring Scheme 2 & 0.0064 & 0.0218 & 0.0341 \\
Deblurring Scheme 3 & 0.0269 & 0.0410 & 0.0669 \\
\hline
\end{tabular}

From Table III, it is shown again that the least squares estimation provides best matches. The source blurry and sharpened images using the least squares estimation (Scheme 2) have more similarities and so one can tell less additional information about another.

\section{E. Discrete Energy in Image Deblurring}

The discrete energy measure also indicates how each of three color level elements is distributed. Its formulation is shown in (12), where $\mathrm{E}(x)$ represents the discrete energy with 256 bins and $p(i)$ refers to the probability distribution function at each of three color levels, which contains the histogram counts. For any constant value of the color level, the energy measure reaches its maximum value of one. The larger energy corresponds to the lower total number of intensity levels and the smaller one corresponds to the higher total number of intensity levels. The discrete energy of the source and deblurred images is shown in Table IV.

$$
\mathrm{E}(x)=\sum_{i=1}^{k} p(i)^{2}
$$

From Table IV, the value differences are not significant due to magnitudes of energy levels, but similar conclusions can still be made as those of discrete entropies. That is, all three deblurring schemes actually enhance image quality. 
TABLE IV: DISCRETE ENERGY IN IMAGE DEBLURRING

\begin{tabular}{lccc}
\hline Discrete Energy & Red Level & Green Level & Blue Level \\
\hline Source Ocean Image & 0.0066 & 0.0093 & 0.0109 \\
Deblurring Scheme 1 & 0.0066 & 0.0092 & 0.0108 \\
Deblurring Scheme 2 & 0.0066 & 0.0092 & 0.0108 \\
Deblurring Scheme 3 & 0.0066 & 0.0091 & 0.0108 \\
Source Mount Image & 0.0136 & 0.0168 & 0.0184 \\
Deblurring Scheme 1 & 0.0135 & 0.0165 & 0.0178 \\
Deblurring Scheme 2 & 0.0136 & 0.0166 & 0.0181 \\
Deblurring Scheme 3 & 0.0135 & 0.0165 & 0.0177 \\
\hline
\end{tabular}

\section{F. Contrast in Image Deblurring}

Image contrast is a metric that indicates variations in the gray level distribution or each of three color level distribution by visual perception. It depicts differences among individual objects and background within the same scope of view. It is formulated as (13) and results are listed in Table V.

$$
\text { Contrast }=\sum_{\mathrm{i}=0}^{\mathrm{M}-1} \sum_{\mathrm{j}=0}^{\mathrm{N}-1} \frac{\left[\mathrm{g}(\mathrm{i}, \mathrm{j})-\mathrm{g}_{A V G}\right]^{2}}{\mathrm{M} \times \mathrm{N}}
$$

where $g(i, j)$ is an intensity in the co-occurrence matrix at the coordinates $i$ and $j$. It illustrates an element at coordinates $i$ and $j$ on an image of the size $\mathrm{M} \times \mathrm{N}$.

TABLE V: CONTRAST IN IMAGE DEBLURRING

\begin{tabular}{lccc}
\hline Contrast & Red Level & Green Level & Blue Level \\
\hline Source Ocean Image & 0.2618 & 0.1131 & 0.0521 \\
Deblurring Scheme 1 & 0.2702 & 0.1196 & 0.0602 \\
Deblurring Scheme 2 & 0.2751 & 0.1226 & 0.0652 \\
Deblurring Scheme 3 & 0.2716 & 0.1208 & 0.0635 \\
Source Mount Image & 0.1679 & 0.1047 & 0.0555 \\
Deblurring Scheme 1 & 0.1704 & 0.1101 & 0.0651 \\
Deblurring Scheme 2 & 0.1797 & 0.1116 & 0.0663 \\
Deblurring Scheme 3 & 0.1780 & 0.1117 & 0.0657 \\
\hline
\end{tabular}

From Table V, for each color level, all three deblurring schemes give rise to better contrast than the blurry source images. When a comparison is made among three deblurring schemes, the least squares estimation generates the best contrast. The contrast is also higher generated by Wiener Filtering than by Blind Deconvolution.

\section{G. Homogeneity in Image Deblurring}

This measure is a direct measure of local homogeneity of a grayscale image, which relates inversely to the image contrast. Higher values of homogeneity measures indicate less structural variations and lower values indicate more structural variations. Larger values are corresponding to higher homogeneity and smaller values are corresponding to lower homogeneity. It is formulated as (14) and the actual results are listed in Table VI:

$$
\text { Homogeneity }=\sum_{i=0}^{\mathrm{M}-1} \sum_{j=0}^{\mathrm{N}-1} \frac{1}{1+(i-j)^{2}} \mathrm{~g}(i, j)
$$

where $g(i, j)$ is an intensity in the co-occurrence matrix at the coordinates $i$ and $j$.

TABLE VI: HOMOGENEITY IN IMAGE DEBLURRING

\begin{tabular}{lccc}
\hline Homogeneity & Red Level & Green Level & Blue Level \\
\hline Source Ocean Image & 0.8789 & 0.9431 & 0.9740 \\
Deblurring Scheme 1 & 0.8780 & 0.9409 & 0.9705 \\
Deblurring Scheme 2 & 0.8773 & 0.9392 & 0.9700 \\
Deblurring Scheme 3 & 0.8779 & 0.9401 & 0.9702 \\
Source Mount Image & 0.9230 & 0.9498 & 0.9774 \\
Deblurring Scheme 1 & 0.9199 & 0.9460 & 0.9675 \\
Deblurring Scheme 2 & 0.9130 & 0.9449 & 0.9619 \\
Deblurring Scheme 3 & 0.9141 & 0.9452 & 0.9627 \\
\hline
\end{tabular}

From Table VI, for each color level, all three deblurring schemes reduce the homogeneity with sharper images. The best results come from the least squares estimation, which is followed by Wiener filtering and blind deconvolution.

\section{CONCLUSION}

For digital aerial imaging based pattern recognition, uncertainties and disturbances are unavoidable. In order to reduce the blurring effects of aerial images stem from the atmospheric medium dispersing and object moving, instead of employing high computation cost deblurring schemes, this paper presents several classical and efficient approaches in image deblurring, better results are presented with a little effort of scientific computing. To evaluate the results quantitatively, information metrics are proposed based on the information theory, where the discrete entropy, relative entropy, discrete energy, mutual information, contrast and homogeneity are all applied. For the true color ocean and land images, each components of true color levels of red, green and blue are analyzed using these metrics. From three color level cases of two source blurry images, it is demonstrated that all 3 proposed schemes work well on providing more detailed information of the actual scene. It is also concluded that via the simple least squares optimization scheme, similarities between blurry and sharpened images are higher than those deblurring schemes with filtering techniques exclusively.

\section{REFERENCES}

[1] R. Gonzalez and R. Woods, Digital Image Processing, 3rd Edition, Prentice-Hall, 2007

[2] S. Haykin, Neural Networks - A Comprehensive Foundation, 2nd Edition, Prentice Hall, 1999 
[3] R. Duda, P. Hart, and D. Stork, Pattern Classification, 2nd Edition, John Wiley and Sons, 2000

[4] D. MacKay, Information Theory, Inference and Learning Algorithms, University of Cambridge Press, 2005

[5] Z. Ye and G. Auner, "Linear Filtering and Nonlinear Fuzzy Logic Filtering for Sample Identification with Raman Spectroscopy," in Proceedings of the 2003 IEEE International Conference on Systems, Man and Cybernetics (SMC 2003), pp. 4619-24, Oct. 5-8, 2003, Washington, DC, USA

[6] T. Li and L. Li, "A Joint Estimation Approach for Two-tone Image Deblurring by Blind Deconvolution," IEEE Transactions on Image Processing, vol.11, pp. 847-58, Aug. 2002.

[7] V. Katkovnik, K. Egiazarian, and J. Astola, "A Spatially Adaptive Nonparametric Regression Image Deblurring," IEEE Transactions on Image Processing, vol. 14, pp. 1469-78, Oct. 2005.

[8] R. Vio, J. Nagy, and L. Tenorio, "A Simple But Efficient Algorithm for Multiple-image Deblurring," Astronomy \& Astrophysics, vol. 416, no. 1, pp. 403-10, March 2004

[9] D. Pierre1, D. Sylvain, F. Jacques, and R. Bernard, “A Best Wavelet Packet Basis for Joint Image Deblurring-Denoising and Compression," in Proceedings of SPIE - Mathematics of Data/Image Coding, Compression, and Encryption V, with Applications, vol. 4793, pp. 242-253, July $9-10$, 2002,

[10] A. Jalobeanu, L. Blanc, and J. Zerubia, "Satellite Image Deblurring Using Complex Wavelet Packets," International Journal of Computer Vision, vol. 51, no. 3, pp. 205-217, 2003

[11] W. Wang, X. Yi, and P. Fei, "Denoising of SAR Images Based on Lifting Scheme Wavelet Packet Transform," Geo-spatial Information Science, vol. 11, no. 4, pp. 257-261.

[12] A. Toumi, A. Taleb-Ahmed, K. Benmahammed, and N. Rechid, "Particle Swarm Optimization for Image Deblurring," in Proc. First Mediterranean Conference on Intelligent Systems and Automation, Algeria, pp. 454-459, 2008.

[13] Z. Ye, "Artificial Intelligence Approach for Biomedical Sample Characterization Using Raman Spectroscopy," IEEE Transactions on Automation Science and Engineering, vol. 2, issue 1, pp. 67-73, January 2005

[14] Z. Ye, H. Mohamadian, and Y. Ye, "Discrete Entropy and Relative Entropy Study on Nonlinear Clustering of Underwater and Arial Images," in Proceedings of the 2007 IEEE International Conference on Control Applications (CCA 2007), Singapore, Oct. 1-3, 2007, pp. 318-323.

[15] Z. Ye, H. Cao, S. Iyengar, and H. Mohamadian, "Medical and Biometric System Identification for Pattern Recognition and Data Fusion with Quantitative Measuring," Systems Engineering Approach to Medical Automation, Chapter Six, Artech House Publishers, pp. 91-112, October, 2008

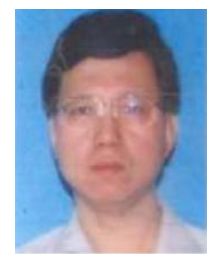

Zhengmao Ye received the B.E. degree from Tianjin University and the first M.S. degree from Tsinghua University, China; the second M.S. and $\mathrm{Ph} . \mathrm{D}$ degrees in electrical engineering from Wayne State University, USA. He is a Senior Member of IEEE and the Founder and Director of Systems and

Control Laboratory at Southern University. Currently Dr. Ye serves as an Associate Professor in Department of Electrical Engineering, Southern University at Baton Rouge, LA 70808, USA.

Dr. Ye's research interests include modeling, control and optimization with diverse applications on electrical, mechanical, automotive and biomedical systems, as well as signal processing and image processing. Dr. Ye is the first multi-disciplinary researcher who has the first author publications covering all the leading control proceedings in three most prestigious engineering societies (IEEE, ASME, SAE), specifically, IEEE (CDC, CCA, SMC, ACC, ISIC, FUZZ, IJCNN, CEC, CASE, ICCA, SOSE, MSC Congress, WCCI Congress), ASME (IMECE Congress, ICES, JRCICE), SAE (USA Congress, EAEC Congress, PFL Congress). He also holds Sole Authorships in IEEE Transactions and SAE Transactions as well as the First Authorships in top tier international journals. He has been an academic reviewer for over 160 articles submitted to the IEEE, ASME, SAE and numerous international journals. Dr. Ye was the recipient of the Chinese National Fellowship (First Prize) at Tianjin University, the USA Allied Signal Fellowship (First Prize) at Tsinghua University and the Most Outstanding Faculty of Electrical Engineering Department at Southern University. He was selected for inclusion in Marquis Who's Who in 2008 .

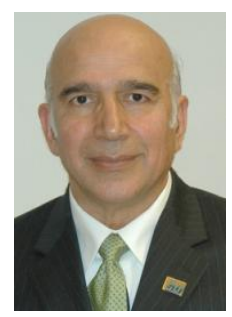

Habib Mohamadian serves as the Professor and Dean, College of Engineering, Southern University at Baton Rouge, Louisiana, USA. He received the B.S. degree from University of Texas at Austin, M.S. and Ph.D. degrees in College of Engineering from Louisiana State Univ. His research interests include various aspects of engineering education and practice. The Dean oversees the College's strategic planning, program development, academic affairs, government and industry relations, and research initiatives. 